\title{
Shock response of nanotwinned copper from large-scale molecular dynamics simulations
}

\author{
Fuping Yuan* and Xiaolei $\mathrm{Wu}^{\dagger}$ \\ State Key Laboratory of Nonlinear Mechanics Institute of Mechanics, Chinese Academy of Science, Beijing 100190, \\ People's Republic of China
}

(Received 24 May 2012; revised manuscript received 19 July 2012; published 10 October 2012)

\begin{abstract}
A series of large-scale molecular dynamics simulations have been performed to investigate the shock response of nanotwinned (NT) $\mathrm{Cu}$, including shock-induced plasticity, strength behind the shock front, and spall behaviors. In this study, two configurations were investigated at an impact velocity of $600 \mathrm{~m} / \mathrm{s}$, i.e., the practical NT polycrystalline $\mathrm{Cu}$ with an average grain size of $10 \mathrm{~nm}$ and the simple NT single-crystalline $\mathrm{Cu}$ with an impact direction of [112] ]. In the NT polycrystalline $\mathrm{Cu}$, the average flow stress behind the shock front first increases with decreasing twin-boundary spacing (TBS), reaching a maximum at a critical TBS, and then decreases as the TBS become even smaller. This trend of the average flow stress with decreasing TBS is due to two competitive dislocation activities under shock loading, with one being inclined to the twin boundaries (the dislocation-twin boundary intersecting) and the other parallel to the twin boundaries (detwinning with twin-boundary migration). Since voids always nucleate near the grain boundary (GB) junctions and then grow along the GBs to create spallation, no apparent correlation between the spall strength and TBS is observed in the NT polycrystalline $\mathrm{Cu}$. However, the spall strengths of the NT single-crystalline $\mathrm{Cu}$ are found to increase with decreasing TBS. Two partial dislocation slips initiated from each twin boundary create voids at the intersections between the partial dislocation slips and twin boundaries. The smaller TBSs result in a larger number of twin boundaries and provide more nucleation sites for voids, requiring a higher tensile stress to create spallation in the NT single-crystalline $\mathrm{Cu}$. These findings should provide insights for understanding the deformation physics of the NT metals subjected to shock loading.
\end{abstract}

DOI: $10.1103 /$ PhysRevB.86.134108

PACS number(s): $61.72 . \mathrm{Mm}, 61.72 . \mathrm{Ff}, 46.40 . \mathrm{Cd}, 25.40 . \mathrm{Sc}$

\section{INTRODUCTION}

The design of impact-tolerant structures demands comprehensive understanding of deformation physics for materials subjected to shock loading. The shock behavior of materials is typically investigated using plate impact experiments, ${ }^{1,2}$ wherein strain rates of $10^{5}-10^{6} \mathrm{~s}^{-1}$ are achieved. However, recent advancements using laser-induced shock compression allow the ability to reach strain rates up to $10^{10} \mathrm{~s}^{-1} \cdot{ }^{3-5}$ Most of the shock experiments are aimed at evaluating the macroscopic mechanical properties, such as the shock front structure, the shock Hugoniot, the strength behind the shock front, and spall strength. However, the dynamic response of materials induced by shock wave loading is often strongly dependent on microstructure evolutions and the underlying physics of deformation mechanisms. These deformation mechanisms at an atomic level can be investigated using molecular dynamics (MD) simulations. Moreover, MD simulations are also a proven tool in modeling shock response and providing valuable physical insights for experimental results conducted at extreme conditions. ${ }^{6-27}$

Previous MD studies on the shock response of $\mathrm{Cu}$ were mostly limited on either single-crystal or twin-free nanocrystalline (NC) metals. ${ }^{6-10,12,13,17-20,22,27}$ Germann et al. ${ }^{6,7}$ and Bringa et al. ${ }^{9}$ studied the shock propagation along the low index directions of [001], [110], and [111] for single-crystal Cu by using Lennard-Jones (LJ) potentials and embedded atom method (EAM) potentials, respectively. The shock-induced plasticity from their results was quite similar qualitatively. Their results indicated that the plastic wave was always overdriven (i.e., no elastic precursor observed) when the shock wave was traveling along the [001] direction, while an elastic precursor separated from the plastic region at the shock front was observed when the shock wave was traveling along the directions of [110] and [111]. MD simulations have been also employed to study the micromechanisms associated with void growth and coalescence at the spall plane for single-crystal $\mathrm{Cu}$ subjected to shock loading. ${ }^{8,12,18,19,22,27}$ Their simulation results showed that the first traversal of the shock wave created various kinds of defects in the materials, and voids were formed at stacking fault intersections during the subsequent traversal of the released tensile waves reflected from free surfaces. In contrast to single crystal, the presence of a large volume fraction of grain boundaries (GBs) in the $\mathrm{NC}$ metals can be expected to have a significant effect on the mechanical properties and microstructure evolutions under shock loading. For example, the void nucleation was found to occur along GBs at the spall plane in $\mathrm{NC} \mathrm{Cu}$, and the spall strength of $\mathrm{NC} \mathrm{Cu}$ at similar strain rates was found to be much smaller compared to that of single-crystal $\mathrm{Cu} .{ }^{12,20,22}$ Dongare et al. ${ }^{22}$ have investigated the effect of impact velocity on the spall behavior of $\mathrm{NC} \mathrm{Cu}$ using MD simulations. It was found that higher impact velocities resulted in a higher strain rate, higher values of spall strengths, and a larger number of voids in smaller times. Bringa et al. ${ }^{10}$ have studied the pressure effect on the shock compression of $\mathrm{NC} \mathrm{Cu}$. An ultrahigh strength behind the shock front was observed due to the high pressure and the suppression of GBs sliding under shock loading. As pressure increased, a shift in the maximum strength to lower grain sizes was observed due to the suppression of GB associated plasticity. However, beyond a critical pressure, higher temperatures due to adiabatic heating resulted in a drop in strength.

The nanotwinned (NT) $\mathrm{Cu}$ shows both high strength and high ductility compared to conventional coarse-grained polycrystalline $\mathrm{Cu}^{28,29}$ Uniform uniaxial deformation of NT $\mathrm{Cu}$ has been investigated previously using both experiments and MD simulations. ${ }^{30-44}$ Interestingly, the strength of such NT $\mathrm{Cu}$ was found to first increase with decreasing twin-boundary 
spacing (TBS), reaching a maximal strength at a critical twin thickness, then decrease with further reduced twin thickness due to a transition of deformation mechanisms from the classical Hall-Petch type strengthening to a dislocation-nucleationcontrolled softening with twin-boundary migration. ${ }^{29,41}$ Harder and tougher materials under shock loading could offer novel applications, such as improved armor materials. So, it is highly desirable to examine the microstructure evolutions and underlying physics of deformation mechanisms for $\mathrm{NT} \mathrm{Cu}$ subjected to shock loading since it has the potential to achieve both high strength and toughness under shock conditions. In this regard, the focus of this paper is to understand the effect of TBS on the strength behind the shock front, spall strength, and related atomic-level deformation mechanisms in $\mathrm{NT} \mathrm{Cu}$ subjected to shock loading using MD simulations.

\section{COMPUTATIONAL METHODS}

The MD simulations were carried out using the Large-scale Atomic/Molecular Massively Parallel Simulator (LAMMPS) code and a $\mathrm{Cu}$ EAM potential developed by Mishin et al. ${ }^{45}$ This potential was calibrated according to the $a b$ initio values of stacking fault and twin formation energies. In this study, two configurations were considered, i.e., the practical NT polycrystalline $\mathrm{Cu}$ and the simple NT single-crystalline $\mathrm{Cu}$. The initial three-dimensional polycrystalline $\mathrm{Cu}$ samples $(32 \times 32 \times 80 \mathrm{~nm})$ with an average grain size of $d=10 \mathrm{~nm}$ were created using the Voronoi polyhedral construction, including both high and low angle GBs in a random selection of grain orientations. In each grain, twins were inserted by mirroring a portion of the matrix with respect to a twin plane. The same Voronoi grain structure and the same crystallographic orientations of all grains were retained as the TBS changes. Seven samples with initial uniform TBSs of $\lambda=0.63,0.83$, $1.04,1.25,1.67,3.13$, and $4.17 \mathrm{~nm}$ were simulated in order to investigate the TBS effect on the shock response of NT polycrystalline $\mathrm{Cu}$. Preparations for NT single-crystalline $\mathrm{Cu}$ $(32 \times 32 \times 80 \mathrm{~nm})$ were relatively straightforward. The impact direction is [112] , while the transverse directions are [111] and [110]. Since the spall behavior will be expected to show no large difference as long as the TB is parallel to the impact direction, the results with the impact direction along [110] will not be shown in the current study. For NT single-crystalline $\mathrm{Cu}$, five samples with different twin spacings $(\lambda=0.63,0.83$, $1.04,2.08$, and $4.17 \mathrm{~nm}$ ) were considered. Periodic boundary conditions were imposed along transverse directions ( $X$ and $Y$ directions) to mimic one-dimensional (1D) strain shock loading, while the system was kept free in the shock loading direction ( $Z$ direction). Before shock loading, the as-created samples were first subjected to energy minimization by the conjugate gradient method, then gradually heated up to the desired temperature in a stepwise fashion, and finally relaxed in the Nose-Hoover isobaric-isothermal ensemble (NPT) under both a pressure of 0 bars and a desired temperature $(1 \mathrm{~K})$ for 200 ps. The local atomic arrangement of the deformed configuration was then visualized using the local crystalline order method. ${ }^{46}$ The relaxed NT polycrystalline and singlecrystalline $\mathrm{Cu}$ systems with a TBS of $1.04 \mathrm{~nm}(\sim 7000000$ atoms) are shown in Figs. 1(a) and 1(b) respectively, with atoms colored according to common neighbor analysis (CNA)
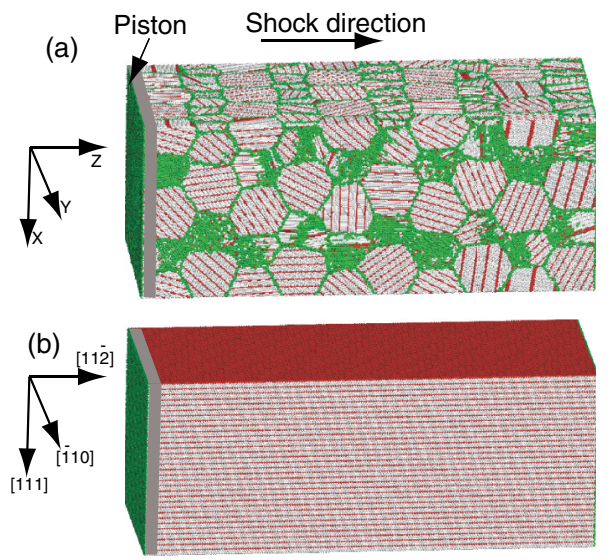

FIG. 1. (Color online) (a) The relaxed NT polycrystalline $\mathrm{Cu}$ system with a TBS of $1.04 \mathrm{~nm}$. (b) The relaxed NT single-crystalline $\mathrm{Cu}$ system with a TBS of $1.04 \mathrm{~nm}$. The atoms are colored according to CNA values. The gray color stands for perfect fcc atoms, red stands for hcp atoms, and green stands for GBs, dislocation core, free surface, and other atoms. The same color coding based on CNA values is used in all figures of the present study.

values. The gray color stands for perfect fcc atoms, red stands for hcp atoms, and green stands for GBs, dislocation core, free surface, and other atoms. The same CNA color coding is used in the following figures.

To achieve shock-induced deformation, the first $2 \mathrm{~nm}$ of the sample was chosen as the piston and the atoms in the piston were given an impact velocity $U_{p}$ in the shock direction ( $Z$ direction). MD simulations of shock wave propagation were performed using a pulse with a sufficiently long duration (14 ps), while a square pulse of 8 ps was used to induce spall at the prescribed region. Shock simulations adopt the microcanonical NVE ensemble. The time step for integrating the equations of motion was 2 fs. An impact velocity of $600 \mathrm{~m} / \mathrm{s}$ was chosen here, at which the NT polycrystalline shows plasticity under shock compression due to GBs while the NT single-crystalline $\mathrm{Cu}$ remains elastic upon shock compression. ${ }^{20}$ This impact velocity was also found to generate enough tensile stress to induce spall in both the NT polycrystalline and single-crystalline $\mathrm{Cu} .{ }^{22}$ We divided the simulation cell into fine bins only along the shock direction (the $1 \mathrm{D}$ binning analysis) by neglecting the heterogeneities in the transverse directions, and the average physical properties were obtained in each bin, such as particle velocity $\left(U_{p}\right)$ and stress $\left(\sigma_{i j}\right)$. The center-of-mass velocity of a bin was removed when calculating $\sigma_{i j}$ from the decomposed virial method. ${ }^{20,47}$ The free surface velocity $\left(U_{\mathrm{fs}}\right)$ versus time profile $(t)$ was obtained from the particle velocity evolution at the target free surface. In order to calculate the strength behind the shock front and study the pressure evolution during the spall process, we defined the maximum shear stress $2 \tau_{\max }$ as $2 \tau_{\max }=\sigma_{z z}-\left(\sigma_{x x}+\sigma_{y y}\right) / 2$ and the hydrostatic pressure $P$ as $P=-\left(\sigma_{x x}+\sigma_{y y}+\sigma_{z z}\right) / 3$.

\section{SHOCK RESPONSE OF NANOTWINNED POLYCRYSTALLINE Cu}

In shock loading, the piston is driven inward along the impact direction at a constant velocity $U_{p}$, leading to a shock 

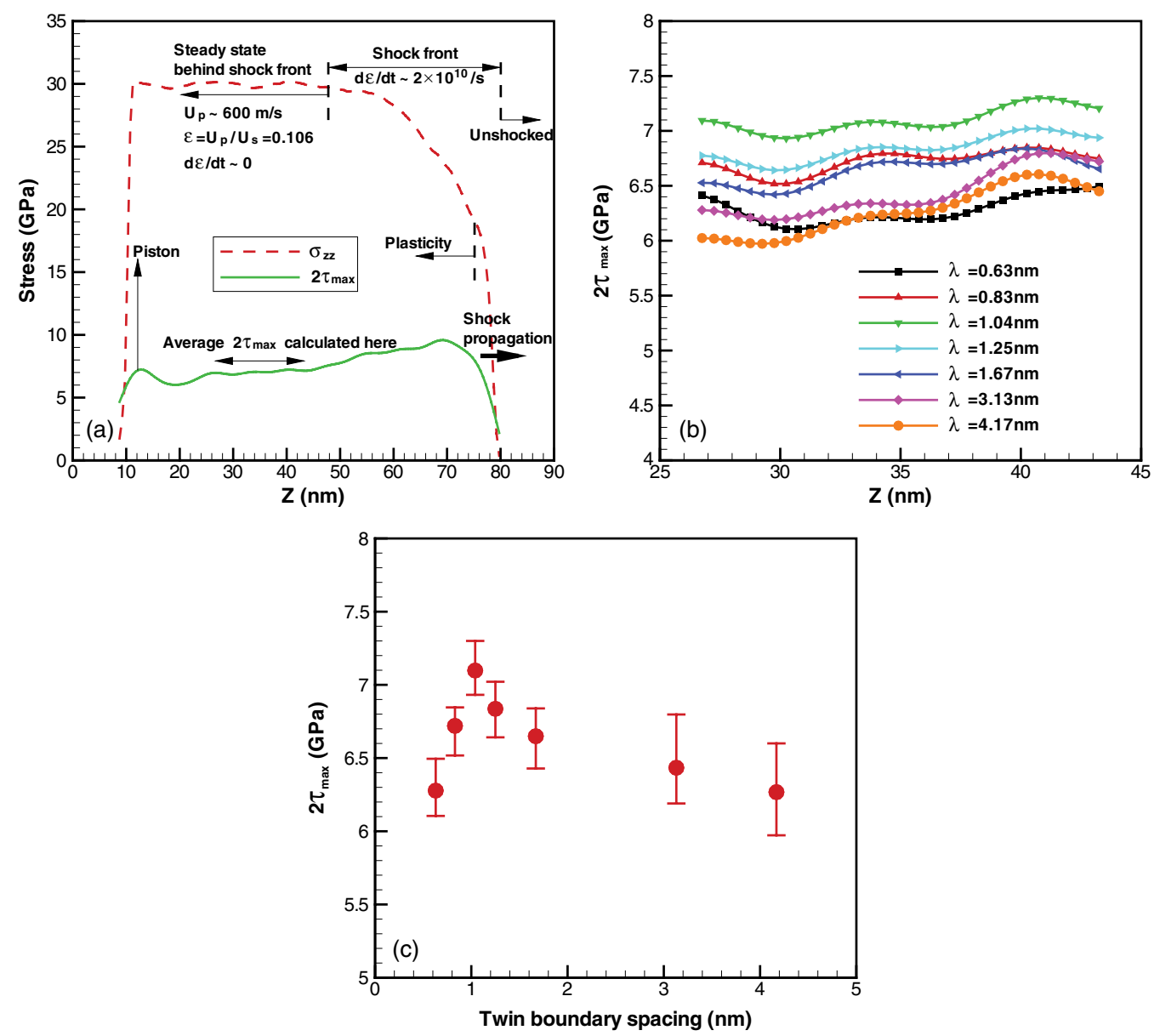

FIG. 2. (Color online) (a) Typical stress $\left(\sigma_{z z}\right.$ and $\left.2 \tau_{\max }\right)$ profiles along the impact direction for the NT polycrystalline Cu with a TBS of $1.04 \mathrm{~nm}$. (b) The flow stress $\left(2 \tau_{\max }\right)$ profiles in the steady region for various NT polycrystalline $\mathrm{Cu}$ with different TBSs. (c) The average flow stress $\left(2 \tau_{\max }\right)$ vs the twin-boundary spacing for the NT polycrystalline $\mathrm{Cu}$. Error bars represent the standard deviation from statistical analysis in the steady region.

wave with velocity $U_{s}$. The major difference between shock compression and homogeneous compression is that the strain and stress in the shock front are inhomogeneous and the strain rate in the shock front is very high. ${ }^{10}$ However, the total volumetric strain behind the shock front, $\varepsilon$, is constant and determined by $\varepsilon=U_{p} / U_{s}(d \varepsilon / d t=0)$. The stress along the shock direction behind the shock front, $\sigma_{z z}$, is also constant and is given approximately by $\sigma_{z z}=\rho_{0} U_{p} U_{s}$, where $\rho_{0}$ is the density of the preshocked material. The strain rate within the shock front is roughly given by $\varepsilon$ divided by the shock front rise time, i.e., $d \varepsilon / d t \approx \varepsilon U_{s} \Delta_{z}$, where $\Delta_{z}$ is the shock front width. Figure 2(a) shows a typical shock stress profile for the NT polycrystalline $\mathrm{Cu}$ with a TBS of $1.04 \mathrm{~nm}$. Values are obtained from a snapshot of MD simulations at $13 \mathrm{ps}$ after the piston begins to move with $600 \mathrm{~m} / \mathrm{s}$. Portions of samples reached by the shock wave front undergo a fast deformation of $d \varepsilon / d t \approx 2 \times 10^{10} / \mathrm{s}$ during the short shock rise time (several ps) and then remain at constant strain $(\varepsilon=0.106)$, eventually relaxing the shear stress and reaching a steady state.

With $600 \mathrm{~m} / \mathrm{s}$ of $U_{p}, \sigma_{z z}$ for the NT polycrystalline $\mathrm{Cu}$ is approximately $30 \mathrm{GPa}$. The average flow stress behind the shock front (within the steady state region) was calculated via the maximum shear stress, $2 \tau_{\max }=\sigma_{z z}-\left(\sigma_{x x}+\sigma_{y y}\right) / 2$. The maximum shear stress profiles along the $Z$ direction (within the steady state region) for various NT polycrystalline $\mathrm{Cu}$ with different TBSs are shown in Fig. 2(b). Moreover, the average flow stress in the steady state region as a function of TBS for the NT polycrystalline $\mathrm{Cu}$ is shown in Fig. 2(c). It is found that the NT polycrystalline $\mathrm{Cu}$ shows an ultrahigh strength (6.3-7.2 GPa) behind the shock front due to the high shock pressure, as compared to that under uniform uniaxial deformation (2.3-3.4 GPa) at a similar strain rate. ${ }^{41}$ It also can be seen that the average flow stress behind the shock front first increases with decreasing TBS, reaching a maximum at a critical TBS, and then decreases as the twin spacing becomes even smaller, similar to the observations under uniform tensile deformation. ${ }^{29,41}$

In order to understand the trend of the average flow stress behind the shock front as a function of TBS, deformation patterns of two samples based on CNA values with different TBSs $(\lambda=3.13$ and $0.63 \mathrm{~nm})$ are shown in Figs. 3(a) and 3(b), respectively. Only a thin slab along the transverse direction ( $X$ direction, $1 \mathrm{~nm}$ thick) within the steady state region is shown for clarity. The pictures are taken from a snapshot of MD simulations at $13 \mathrm{ps}$ after the piston begins to move with $600 \mathrm{~m} / \mathrm{s}$. From Fig. 3, it can be seen that there exist two types of competitive dislocation activities in addition to the grain-boundary-associated deformation mechanisms under 


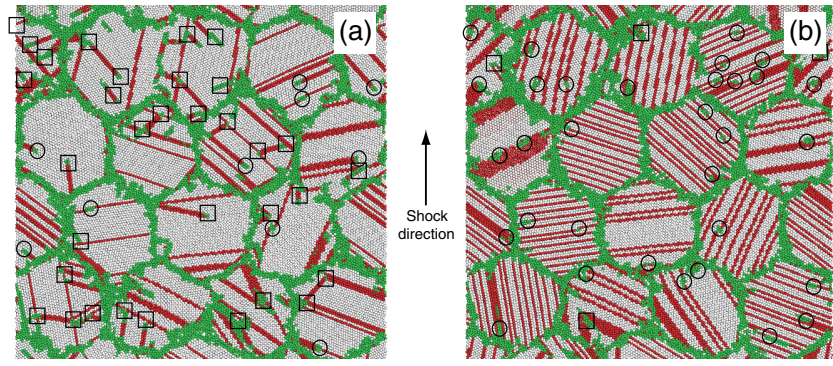

FIG. 3. (Color online) Simulated deformation patterns in the NT polycrystalline $\mathrm{Cu}$ with TBSs of (a) $\lambda=3.13 \mathrm{~nm}$ and (b) $\lambda=0.63 \mathrm{~nm}$. Only a thin slab along the transverse direction ( $1 \mathrm{~nm}$ thick) within the steady state region is shown for clarity. The process by dislocations cutting across twin planes is marked by a black square, while the process by partial dislocations gliding parallel to twin planes is marked by a black circle.

shock loading, with one being inclined to the twin boundaries (marked by a black square) and the other parallel to the twin boundaries leading to detwinning (marked by a black circle). With decreasing TBS, the dominated deformation mechanisms are shifted from cutting through twin planes to detwinning with twin-boundary migration. In the first case, plastic deformation is mediated by partial dislocations (emitted from grain boundaries) cutting into the neighboring twin planes, and the twin-boundary migration plays a much smaller role in accommodating plastic deformation. However, in the latter case, plastic deformation is mediated by partial dislocations (nucleated from grain boundaries) gliding along the twin planes, and the dislocation intersecting is rarely observed here. So the first increasing trend of the average flow stress with decreasing TBS is due to the classical Hall-Petch type strengthening, i.e., dislocation cutting through twin planes, while the decreasing trend of the average flow stress with an even smaller TBS should be related to a dislocation-nucleation-controlled softening with twin-boundary migration. ${ }^{41}$

\section{SPALL BEHAVIOR OF NANOTWINNED POLYCRYSTALLINE Cu}

In order to understand the temporal response of the NT polycrystalline $\mathrm{Cu}$ subjected to a square pulse of shock loading (8 ps duration), the typical stress ( $P$ and $\sigma_{z z}$ ) files along the impact direction at various times for the sample with a TBS of $1.04 \mathrm{~nm}$ are shown in Fig. 4. In the present paper, a positive value corresponds to compressive stress, while a negative value corresponds to tensile stress. First, the sample is compressed by the shock compression wave, reaching a steady state with stresses of $\sigma_{z z} \sim 30 \mathrm{GPa}$ and $P \sim 25 \mathrm{GPa}$ at $12 \mathrm{ps}$. The compressed material moves freely as the shock wave reaches the rear free surface [Fig. 4(b)] and reflects as a tensile wave.
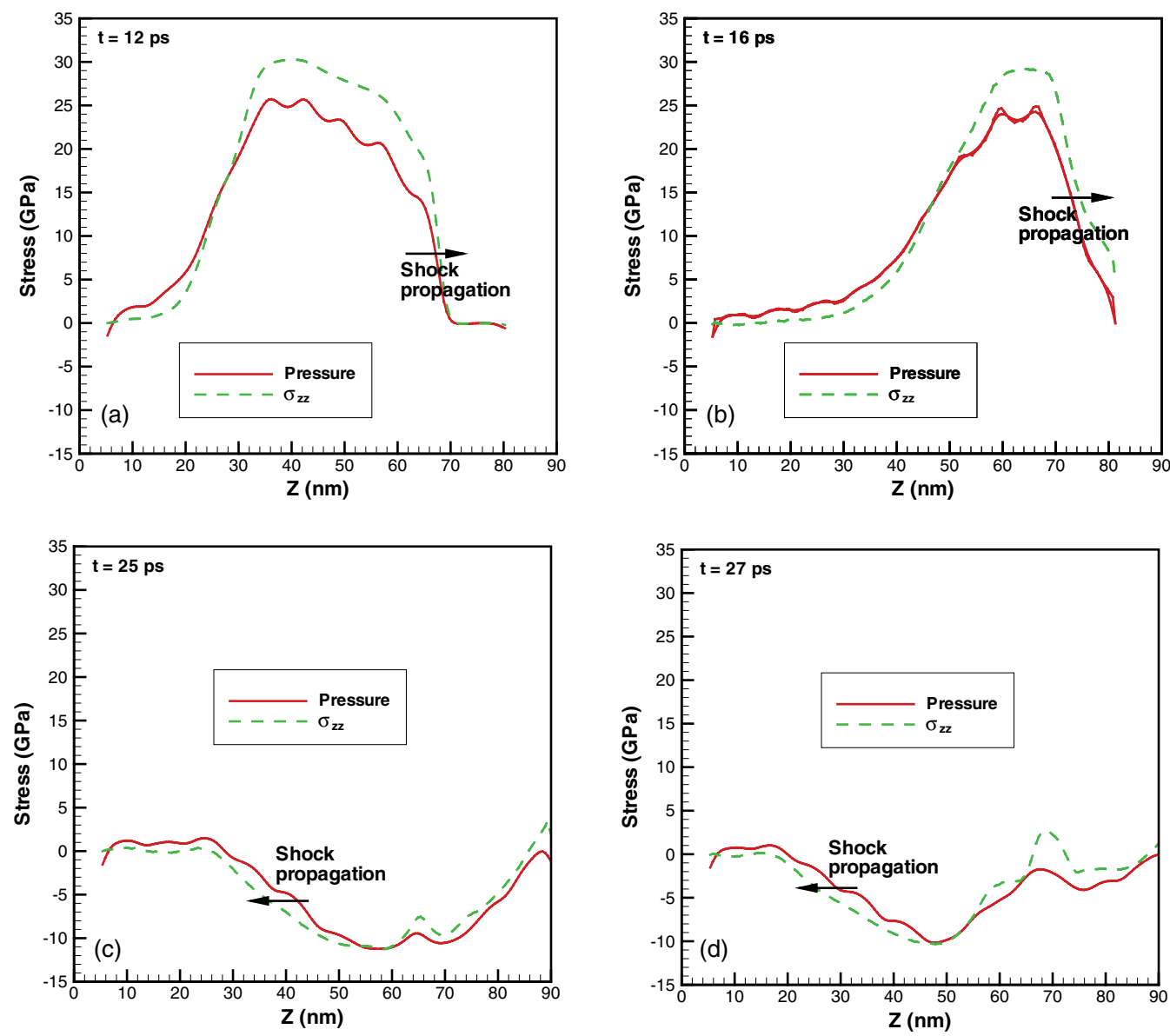

FIG. 4. (Color online) Shock stress profiles as a function of the $Z$ coordinate for the NT polycrystalline Cu with a TBS of $1.04 \mathrm{~nm}$ subjected to a square pulse at a time of (a) $t=12 \mathrm{ps,} \mathrm{(b)} t=16 \mathrm{ps,} \mathrm{(c)} t=25 \mathrm{ps}$, and (d) $t=27 \mathrm{ps}$. 

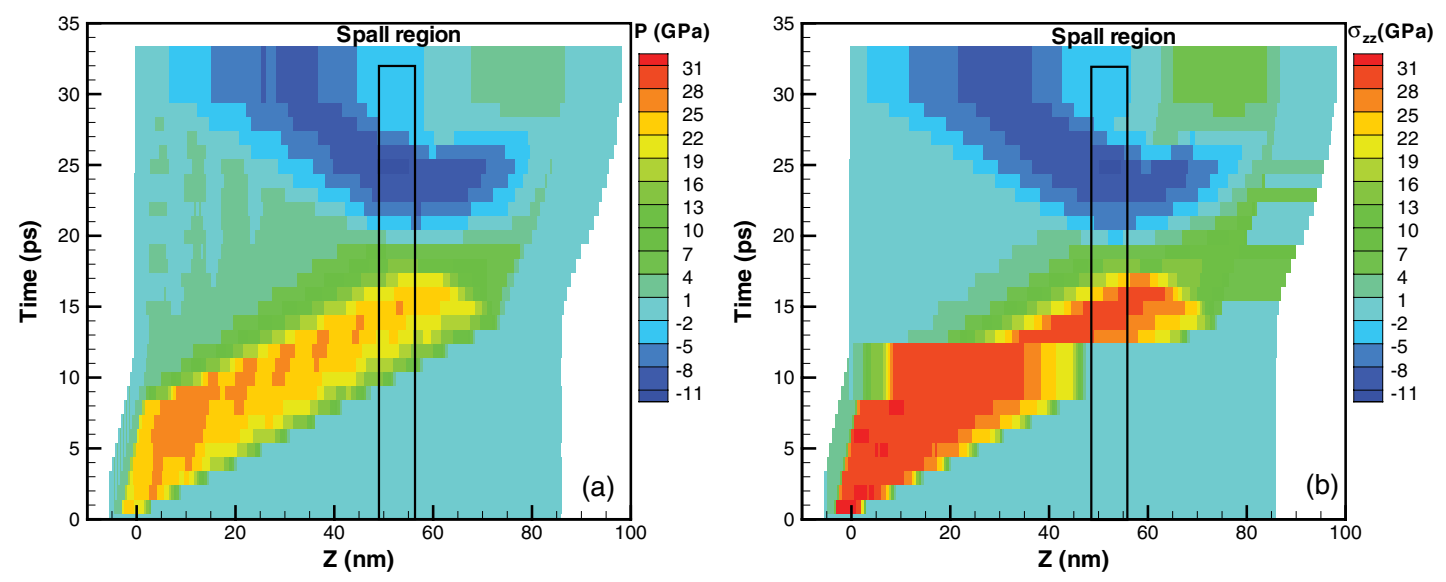

FIG. 5. (Color online) Distribution of (a) pressure and (b) $\sigma_{z z}$ along the shock direction as a function of time for the NT polycrystalline Cu with a TBS of $1.04 \mathrm{~nm}$.

The reflected tensile wave interacts with the tail of the initial compressive wave, resulting in a tensile stress state at $25 \mathrm{ps}$ [Fig. 4(c)]. The tensile wave continues to propagate toward the left at 27 ps [Fig. 4(d)]. It is shown that the material at the prescribed region undergoes a tensile stress state for several ps. Spallation occurs in the material when the tensile stress is larger than the spall strength.

The contour plots of the distribution of pressure $(P)$ and normal stress $\left(\sigma_{z z}\right)$ along the shock direction, as a function of time for the NT polycrystalline $\mathrm{Cu}$ with a TBS of $1.04 \mathrm{~nm}$, are
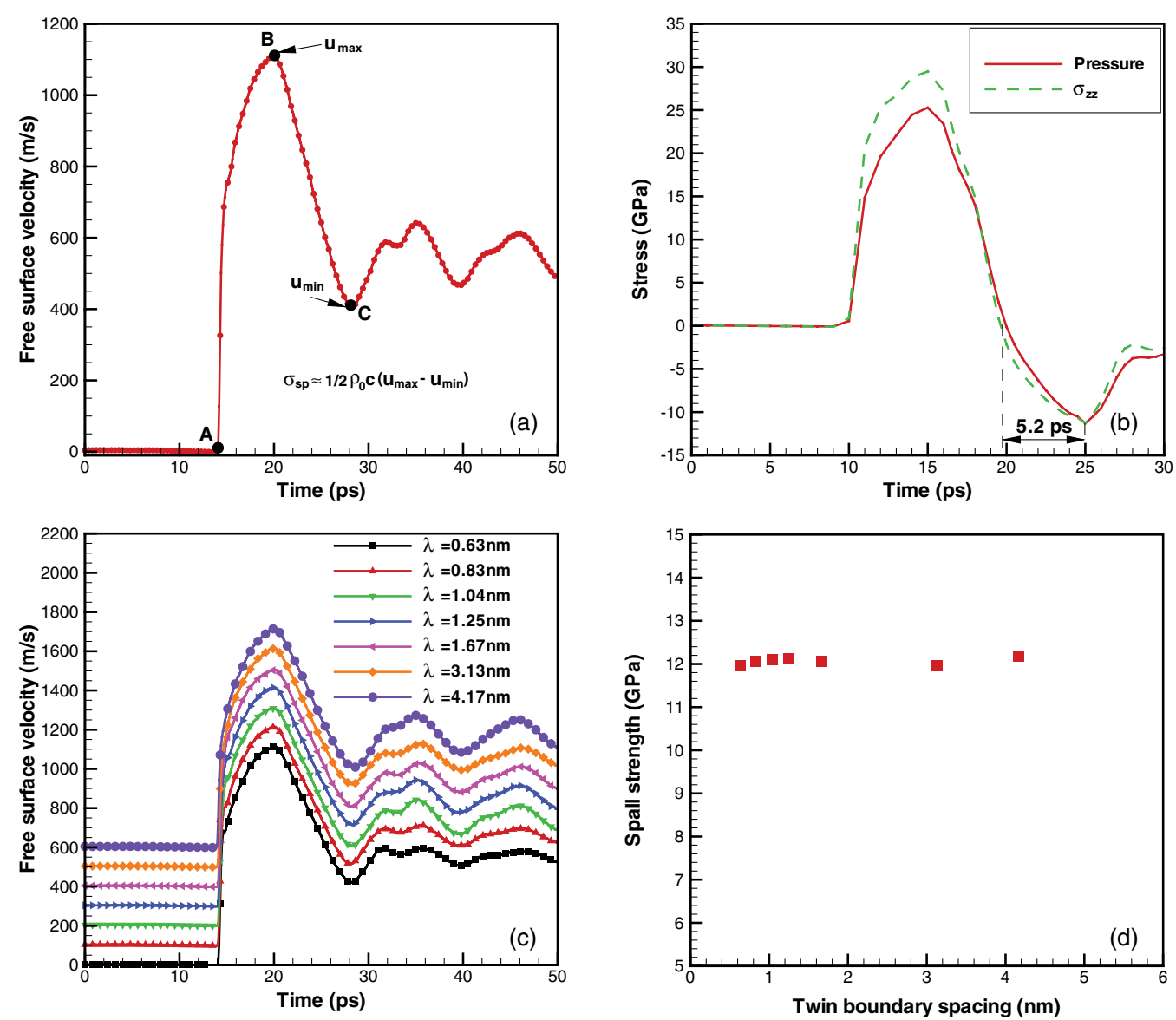

FIG. 6. (Color online) (a) The typical rear free surface velocity profile as a function of time for the NT polycrystalline Cu with a TBS of $1.04 \mathrm{~nm}$ showing the spall pull-back signal and the calculation of spall strength. (b) The evolution of pressure and $\sigma_{z z}$ at the spall plane as a function of time for the NT polycrystalline $\mathrm{Cu}$ with a TBS of $1.04 \mathrm{~nm}$. (c) The rear free surface velocity profiles as a function of time for various NT polycrystalline $\mathrm{Cu}$ with different TBSs (the free surface velocity curves have been shifted toward the upper region by 100 , 200, $300,400,500$, and $600 \mathrm{~m} / \mathrm{s}$ for the samples with TBSs of $0.83,1.04,1.25,1.67,3.13$, and $4.17 \mathrm{~nm}$, respectively, to avoid curve overlapping of similar shapes). (d) The spall strength vs the twin-boundary spacing for the NT polycrystalline $\mathrm{Cu}$. 
shown in Figs. 5(a) and 5(b), respectively. From the figure, it is clearly seen that the spall region undergoes a change of stress states $\left(P\right.$ and $\left.\sigma_{z z}\right)$ from the maximum compression states at $15 \mathrm{ps}$ to the maximum tensile states at $25 \mathrm{ps}$.

The rear free surface velocity is plotted as a function of time for NT polycrystalline $\mathrm{Cu}$ with a TBS of $1.04 \mathrm{~nm}$ in Fig. 6(a). The first shock wave reaches the rear free surface at the time of point A. This shock wave accelerates the material to approximately twice the particle velocity behind the shock front, as indicated by point $B$. The acceleration causes the material to go into a tension state as a result of which the rear free surface velocity decelerates, as indicated by the curve after point $\mathrm{B}$. The tensile stress state causes the voids to nucleate at the spall region, and the nucleation of voids results in a second shock wave causing the acceleration of the rear free surface after point $\mathrm{C}$. This is the so-called typical pull-back signal for spallation.

Within the acoustic approximation, the spall strength can be deduced from the free surface velocity $U_{\mathrm{fs}}$ as $\sigma_{\mathrm{sp}} \approx \frac{1}{2} \rho_{0} c \Delta U_{\mathrm{fs}}=\frac{1}{2} \rho_{0} c\left(U_{\mathrm{max}}-U_{\min }\right) .{ }^{18}$ Here $\rho_{0}$ is the density of the preshocked material, $U_{\max }$ and $U_{\min }$ are the maximum and minimum velocities defined at $\mathrm{A}$ and $\mathrm{B}$ points in Fig. 6(a), and $c$ is a longitudinal or bulk sound speed at zero pressure. For simplicity, we perform linear fitting to the shock velocity-piston velocity results (based on a series of simulations) and take the intercept as $c . c$ is about $3.97 \mathrm{~km} / \mathrm{s}$ for a temperature of $1 \mathrm{~K}$. The density of the preshocked NT polycrystalline $\mathrm{Cu}$ is $8.96 \mathrm{~g} / \mathrm{cm}^{3}$. Based on the pull-back signal from the free surface velocity, the spall strength for NT polycrystalline $\mathrm{Cu}$ with a TBS of $1.04 \mathrm{~nm}$ is calculated as $12.1 \mathrm{GPa}$. Based on the 1D binning analysis, the evolution of pressure $(P)$ and normal stress $\left(\sigma_{z z}\right)$ at the spall plane as a function of time is shown in Fig. 6(b). Although the maximum compressive stresses experienced at the spall plane for $P$ and $\sigma_{z z}$ are different, the maximum tensile stresses experienced at the spall plane for $P$ and $\sigma_{z z}$ are almost the same and are as high as those at $11.3 \mathrm{GPa}$ [Fig. 6(b)]. The rate of pressure and normal stress ( $P$ and $\sigma_{z z}$ ) during the tensile stage is as high as $2.17 \mathrm{GPa} / \mathrm{ps}$. It is indicated that the maximum tensile stress state at the spall plane is approximately an equal-triaxial tensile stress state since the pressure and the normal stress is almost the same. Then the spall strength calculated from the pull-back signal is compared to the value directly obtained from the 1D binning analysis. The former method slightly overestimates the spall strength, but the values of the spall strength from both methods are in reasonable accord (12.1 vs $11.3 \mathrm{GPa}$, and the deviation is 6\%). Thus, the acoustic approximation appears to be a reasonable first-order approximation and the pull-back signal can be considered to deduce the spall strength. ${ }^{18}$

The rear free surface velocity profiles as a function of time are shown in Fig. 6(c) for various NT polycrystalline $\mathrm{Cu}$ with TBSs of $\lambda=0.63,0.83,1.04,1.25,1.67,3.13$, and $4.17 \mathrm{~nm}$. Then the spall strengths for the samples with various TBSs are calculated based on the equation of $\sigma_{\mathrm{sp}} \approx \frac{1}{2} \rho_{0} c\left(U_{\max }-U_{\min }\right)$. Moreover, the spall strength as a function of TBS for the NT polycrystalline $\mathrm{Cu}$ is shown in Fig. 6(d). It is found that the spall strengths of the NT polycrystalline $\mathrm{Cu}$ are nearly independent of the twin spacing.

In order to understand the TBS effect on the micromechanisms of void nucleation and growth for the spallation of the NT polycrystalline $\mathrm{Cu}$, snapshots of a thin section $(1 \mathrm{~nm}$
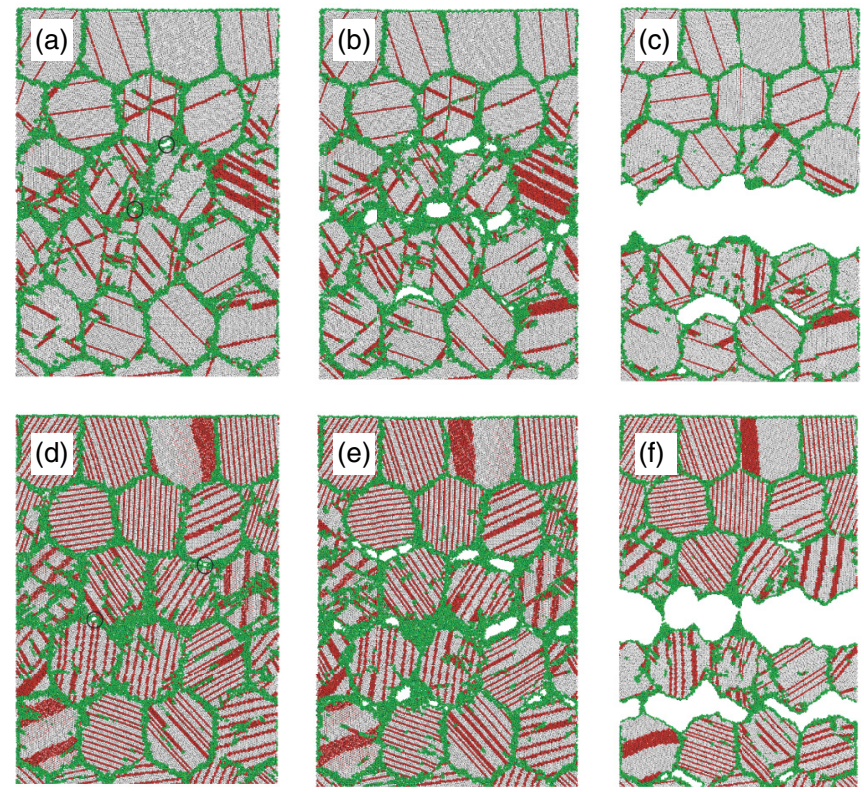

FIG. 7. (Color online) Microstructural evolution of a thin section showing the void nucleation and growth for the NT polycrystalline $\mathrm{Cu}$ with a TBS of $3.13 \mathrm{~nm}$ at a time of (a) $24 \mathrm{ps}$, (b) $32 \mathrm{ps}$, and (c) $40 \mathrm{ps}$ and for the NT polycrystalline $\mathrm{Cu}$ with a TBS of $0.63 \mathrm{~nm}$ at a time of (d) $24 \mathrm{ps,} \mathrm{(e)} 32 \mathrm{ps}$, and (f) $40 \mathrm{ps}$. Only a thin slab along the transverse direction ( $1 \mathrm{~nm}$ thick) including the spall region is shown for clarity. The color coding is based on the CNA values.

thick slab along the $X$ direction, including the spall region) at different times are illustrated in Fig. 7 for the samples with TBSs of $3.13 \mathrm{~nm}$ [Figs. 7(a)-7(c)] and $0.63 \mathrm{~nm}$ [Figs. 7(d)7(f)]. The snapshots show different dislocation behaviors in the grains near the spall region for different TBSs. However, it can be seen that the voids always nucleate near the GB junctions and then grow along the GBs independent of TBS, generating nearly the same spall strength for the NT polycrystalline $\mathrm{Cu}$ with various TBSs [Fig. 6(d)]. The nucleation of voids is mainly attributed to a mechanical separation and sliding of grains at the GBs and not by dislocation behaviors in the grain interior under tensile shock loading. ${ }^{12,20,22}$ The continue tensile shock deformation increases the size of voids, and eventually causes the coalescence of voids and completely spallation. Previous ex situ and in situ quasistatic tensile experiments on nanocrystalline metals ${ }^{48,49}$ also showed that fracture is an intergranular manner and is initiated by the nucleation of voids at GBs and triple junctions through unaccommodated GB sliding. Similar fracture behaviors were also found in MD simulations for other deformation conditions, such as crack propagation in precracked nanocrystalline metals ${ }^{50}$ and triaxial uniform tensile loading in nanocrystalline metals. ${ }^{51}$ Theoretical models ${ }^{52,53}$ indicated that crack initiation at triple junctions is energetically favorable in nanocrystalline metals, and is mediated by the accumulation of the dislocation charge at triple junctions and the accompanied GB sliding. So, in the presence of GBs and triple junctions, the spall strength of the NT polycrystalline $\mathrm{Cu}$ is nearly independent of TBS since GBs and triple junctions are always the energetically favorable sites for void nucleation. 

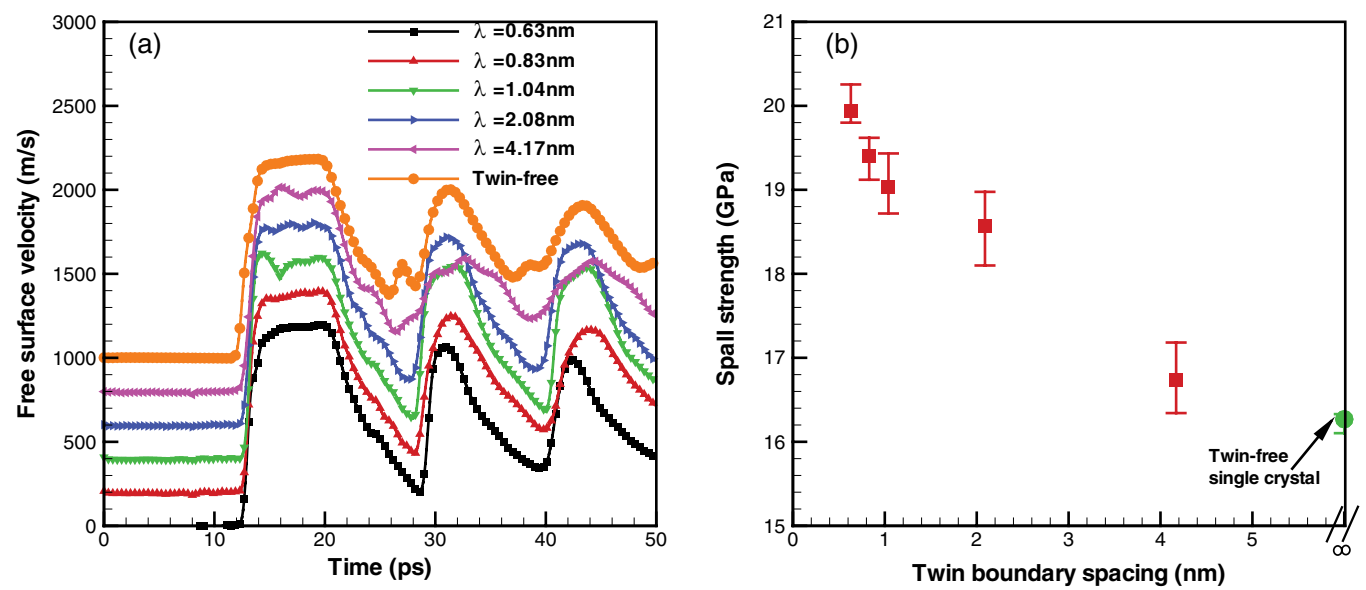

FIG. 8. (Color online) (a) The rear free surface velocity profiles as a function of time for various NT single-crystalline Cu with different TBSs and the twin-free single-crystalline $\mathrm{Cu}$ (the free surface velocity curves have been shifted toward the upper region by $200,400,600,800$, and $1000 \mathrm{~m} / \mathrm{s}$ for the samples with TBSs of $0.83,1.04,2.08$, and $4.17 \mathrm{~nm}$ and the twin-free single-crystalline Cu, respectively, to avoid curve overlapping of similar shapes). (b) The spall strength vs the twin-boundary spacing for the NT single-crystalline Cu. Error bars represent the standard deviation from a statistical analysis in calculating the $U_{\max }$.

\section{SPALL BEHAVIOR OF NANOTWINNED SINGLE-CRYSTALLINE Cu}

It is found in Sec. IV that the spall strength is independent of TBS due to the existence of GBs in the NT polycrystalline $\mathrm{Cu}$. Another intriguing issue worthy of comprehensive study here is how TBS affects the spallation behaviors in NT single-crystalline $\mathrm{Cu}$ without the presence of GBs. In this regard, the TBS effect on the spall strength of NT singlecrystalline $\mathrm{Cu}$ was studied and presented in this section. As indicated in Sec. II, the impact direction is [112]], while the transverse directions are [111] and [1110] for the NT single-crystalline $\mathrm{Cu}$. One twin-free single-crystalline $\mathrm{Cu}$ with the same crystallographic orientations was also simulated for comparison.

The rear free surface velocity profiles as a function of time are shown in Fig. 8(a) for the NT single-crystalline $\mathrm{Cu}$ with TBSs of $\lambda=0.63,0.83,1.04,2.08$, and $4.17 \mathrm{~nm}$ and the twinfree single-crystalline $\mathrm{Cu}$. The spall strengths of the NT singlecrystalline $\mathrm{Cu}$ and the twin-free single-crystalline $\mathrm{Cu}$ were then calculated by the same method as that used in Sec. IV.

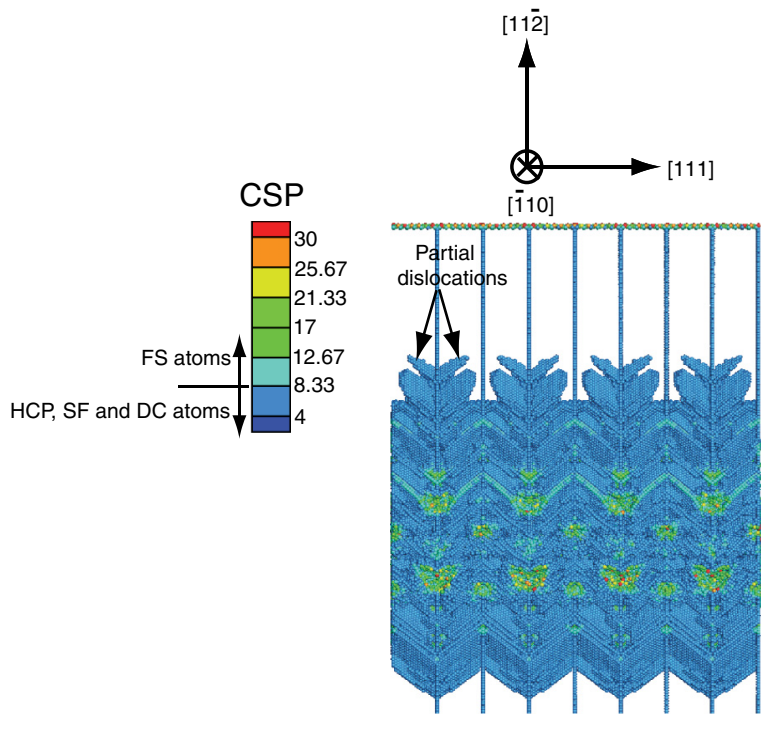

(a)

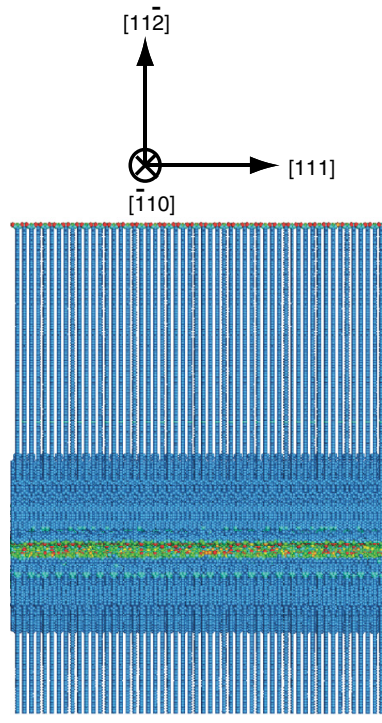

(b)

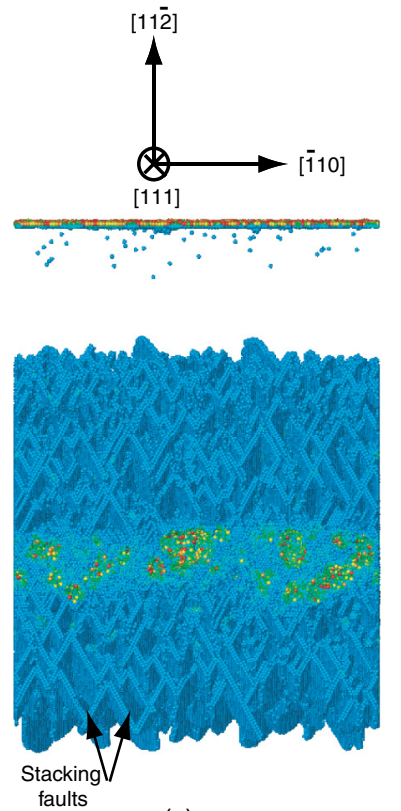

(c)

FIG. 9. (Color online) Void nucleating patterns based on CSP for (a) the NT single-crystalline Cu with a TBS of $4.17 \mathrm{~nm}$, (b) the NT single-crystalline $\mathrm{Cu}$ with a TBS of $0.63 \mathrm{~nm}$, and (c) the twin-free single-crystalline $\mathrm{Cu}$. The colors with smaller CSP values (CSP $<8.33$ ) represent hcp, stacking faults (SFs), and dislocation core (DC) atoms, while the colors with bigger CSP values (CSP $>8.33$ ) represent the free surface atoms or the atoms near the free surface. 
Moreover, the spall strength as a function of TBS for the NT single-crystalline $\mathrm{Cu}$ is shown in Fig. 8(b), where the data for the twin-free single-crystalline $\mathrm{Cu}$ are also included. It is found that the spall strengths of the NT single-crystalline $\mathrm{Cu}$ are higher than those of the twin-free single-crystalline $\mathrm{Cu}$, and increase with decreasing TBS.

In order to understand the trend of spall strength as a function of TBS for the NT single-crystalline $\mathrm{Cu}$, void nucleation patterns based on centrosymmetry parameters (CSPs) for two samples with different TBSs $(\lambda=4.17$ and $0.63 \mathrm{~nm}$ ) are shown in Figs. 9(a) and 9(b), respectively. The deformation pattern for the twin-free single-crystalline $\mathrm{Cu}$ is also shown in Fig. 9(c) for comparison. In Fig. 9, smaller CSP values (CSP $<8.33)$ represent hcp, stacking faults (SFs), and dislocation core atoms, while bigger CSP values (CSP > 8.33) represent the free surface atoms or the atoms near the free surface. For the twin-free single-crystalline $\mathrm{Cu}$, voids are observed to nucleate at stacking fault intersections in the spall region, as shown in Fig. 9(c). This mechanism of void nucleation is very similar to that reported previously. ${ }^{12,14,19,22}$ However, the partial dislocations are observed to initiate at the twin boundaries and propagate at both sides of the twin boundaries in the spall region for the NT single-crystalline $\mathrm{Cu}$ [Fig. 9(a)]. The two partial dislocation slips initiated from each twin boundary create voids at the intersections between the partial dislocation slips and twin boundaries. As indicated in Figs. 9(a) and 9(b), smaller TBSs result in a larger number of twin boundaries and provide more nucleation sites for voids, requiring higher tensile stress to create spallation.

The snapshots of the spallation process based on CNA values for the NT single-crystalline $\mathrm{Cu}$ with s TBS of $4.17 \mathrm{~nm}$ are illustrated in Fig. 10. Voids are observed to initiate at the intersections between the partial dislocation slips and twin boundaries, and the initial voids are of a nonspherical shape in nature [Fig. 10(a)]. Continued tensile deformation increases the size of the voids to create spallation.

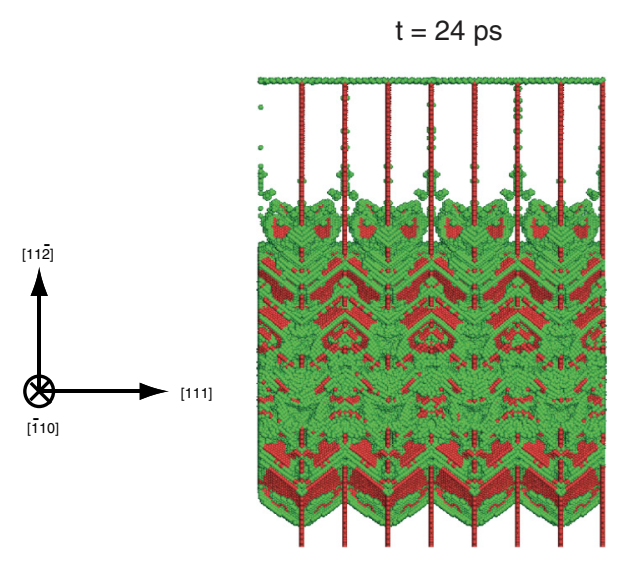

(a)

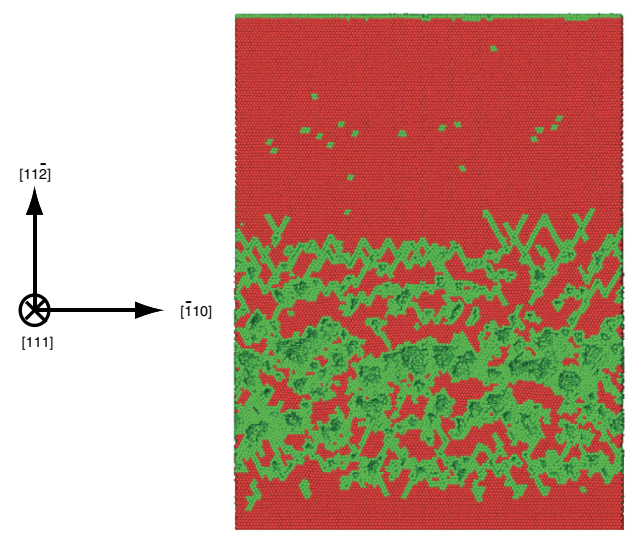

(d)

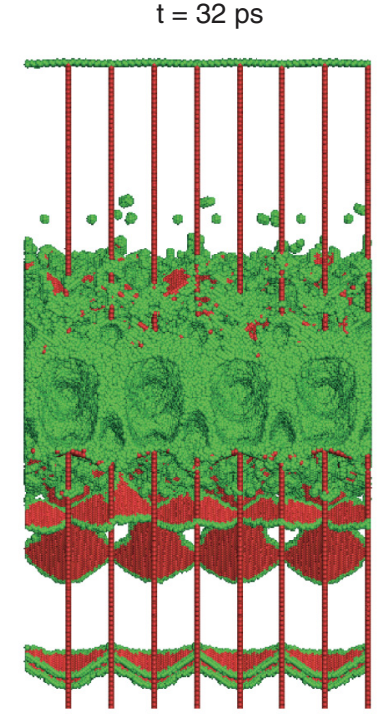

(b)

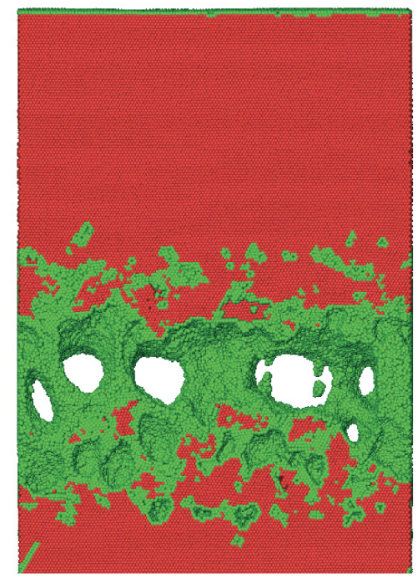

(e)

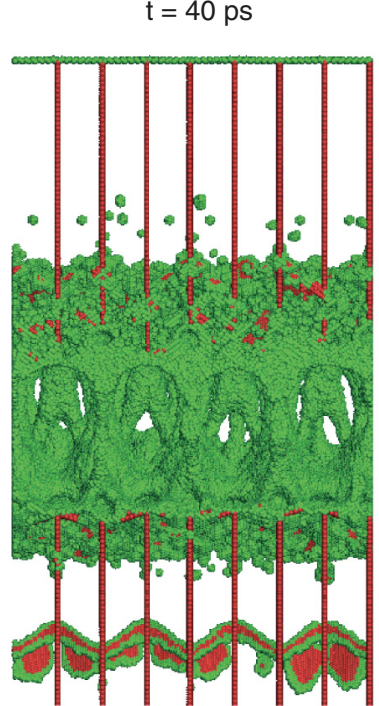

(c)

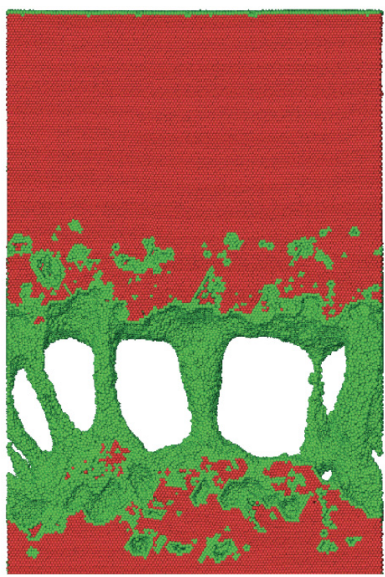

(f)

FIG. 10. (Color online) The snapshots of the spallation process based on CNA values for the NT single-crystalline Cu with a TBS of $4.17 \mathrm{~nm}$. The deformation patterns are observed from the [110] direction at a time of (a) $t=24 \mathrm{ps,} \mathrm{(b)} t=32 \mathrm{ps,} \mathrm{and} \mathrm{(c)} t=40 \mathrm{ps}$. The deformation patterns are observed from the [111] direction at a time of (d) $t=24 \mathrm{ps,} \mathrm{(e)} t=32 \mathrm{ps,} \mathrm{and} \mathrm{(f)} t=40 \mathrm{ps}$. 


\section{CONCLUDING REMARKS}

MD simulations have been carried out to understand the effect of TBS on shock response and the corresponding deformation mechanisms in nanotwinned $\mathrm{Cu}$. The average flow stress behind the shock front first increases with decreasing TBS, reaching a maximum at a critical TBS, and then decreases as the TBSs become even smaller in the NT polycrystalline $\mathrm{Cu}$. This trend is mainly caused by two competitive dislocation activities under shock loading: dislocation-twin boundary intersecting by the inclined dislocations and twin-boundary migration by the parallel dislocations. No apparent correlation between the spall strength and TBS is observed in the NT polycrystalline $\mathrm{Cu}$ since voids always nucleate and grow along the GBs to create spallation. However, TBS has a strong influence on the spall strength of NT single-crystalline $\mathrm{Cu}$, which is found to increase with decreasing TBSs. Voids are found to nucleate at the intersections between twin boundaries and two initiated partial dislocation slips. So the smaller TBSs result in a larger number of twin boundaries and provide more nucleation sites for voids, requiring a higher tensile stress to create spallation in the NT single-crystalline $\mathrm{Cu}$.

\section{ACKNOWLEDGMENT}

The authors would like to acknowledge the financial support of 973 Program of China (Grants No. 2012CB932203, No. 2012CB937500, and No. 2010CB631004) and NSFC (Grants No. 11002151, No. 11222224, and No. 11072243). *fpyuan@lnm.imech.ac.cn

†xlwu@imech.ac.cn

${ }^{1}$ P. Kumar and R. J. Clifton, J. Appl. Phys. 48, 4850 (1977).

${ }^{2}$ F. P. Yuan, V. Prakash, and J. J. Lewandowski, J. Mater. Res. 22, 402 (2007).

${ }^{3}$ H. Tamura, T. Kohama, K. Kondo, and M. Yoshida, J. Appl. Phys. 89, 3520 (2001).

${ }^{4}$ E. M. Bringa, J. U. Cazamias, P. Erhart, J. Stölken, N. Tanushev, B. D. Wirth, R. E. Rudd, and M. J. Caturla, J. Appl. Phys. 96, 3793 (2004).

${ }^{5}$ Y. M. Wang, E. M. Bringa, J. M. McNaney, M. Victoria, A. Caro, A. M. Hodge, R. Smith, B. Torralva, B. A. Remington, C. A. Schuh, H. Jamarkani, and M. A. Meyers, Appl. Phys. Lett. 88, 061917 (2006).

${ }^{6}$ T. C. Germann, B. L. Holian, and P. S. Lomdahl, Phys. Rev. Lett. 84, 5351 (2000).

${ }^{7}$ T. C. Germann, B. L. Holian, P. S. Lomdahl, D. Tanguy, M. Mareschal, and R. Ravelo, Metall. Mater. Trans. A 35A, 2609 (2004).

${ }^{8}$ E. T. Seppälä, J. Belak, and R. E. Rudd, Phys. Rev. B 69, 134101 (2004).

${ }^{9}$ E. M. Bringa, J. U. Cazamias, P. Erhart, J. Stölken, N. Tanushev, B. D. Wirth, R. E. Rudd, and M. J. Caturla, J. Appl. Phys. 96, 3793 (2004).

${ }^{10}$ E. M. Bringa, A. Caro, Y. M. Wang, M. Victoria, J. M. McNaney, B. A. Remington, R. F. Smith, B. R. Torralva, and H. Van Swygenhoven, Science 309, 1838 (2005).

${ }^{11}$ E. M. Bringa, K. Rosolankova, R. E. Rudd, B. A. Remington, J. S. Wark, M. Duchaineau, D. H. Kalantar, J. Hawreliak, and J. Belak, Nat. Mater. 5, 805 (2006).

${ }^{12}$ V. Dremov, A. Petrovtsev, P. Sapozhnikov, and M. Smirnova, Phys. Rev. B 83, 144110 (2006).

${ }^{13}$ B. Cao, E. M. Bringa, and M. A. Meyers, Metall. Mater. Trans. A 38A, 2681 (2007).

${ }^{14}$ S. G. Srinivasan, M. I. Baskes, and G. J. Wagner, J. Appl. Phys. 101, 043504 (2007).

${ }^{15}$ H. N. Jarmakani, E. M. Bringa, P. Erhart, B. A. Remington, Y. M. Wang, N. Q. Vo, and M. A. Meyers, Acta Mater. 56, 5584 (2008).

${ }^{16}$ X. Cui, W. Zhu, H. He, X. Deng, and Y. Li, Phys. Rev. B 78, 024115 (2008).
${ }^{17}$ S. N. Luo, L. Han, Y. Xie, Q. An, L. Zheng, and K. Xia, J. Appl. Phys. 103, 093530 (2008).

${ }^{18}$ S. N. Luo, Q. An, T. G. Germann, and L. Han, J. Appl. Phys. 106, 013502 (2009).

${ }^{19}$ S. N. Luo, T. G. Germann, and D. L. Tonks, J. Appl. Phys. 106, 123518 (2009).

${ }^{20}$ S. N. Luo, T. C. Germann, T. G. Desai, D. L. Tonks, and Q. An, J. Appl. Phys. 107, 123507 (2010).

${ }^{21}$ S. N. Luo, T. G. Germann, D. L. Tonks, and Q. An, J. Appl. Phys. 108, 093526 (2010).

${ }^{22}$ A. M. Dongare, A. M. Rajendran, B. LaMattina, M. A. Zikry, and D. W. Brenner, J. Appl. Phys. 108, 113518 (2010).

${ }^{23}$ W. Ma, W. J. Zhu, and F. Q. Jing, Appl. Phys. Lett. 97, 121903 (2010).

${ }^{24}$ B. Arman, S. Luo, T. C. Germann, and T. Cağin, Phys. Rev. B 81, 144201 (2010)

${ }^{25}$ R. Chau, J. Stölken, P. Asoka-Kumar, M. Kumar, and N. C. Holmes, J. Appl. Phys. 107, 023506 (2010).

${ }^{26}$ J. A. Zimmerman, J. M. Winey, and Y. M. Gupta, Phys. Rev. B 83, 184113 (2011)

${ }^{27}$ S. Rawat, M. Warrier, S. Chaturvedi, and V. M. Chavan, Modell. Simul. Mater. Sci. Eng. 20, 015012 (2012).

${ }^{28}$ L. Lu, Y. Shen, X. Chen, L. Qian, and K. Lu, Science 304, 422 (2004).

${ }^{29}$ L. Lu, X. Chen, X. Huang, and K. Lu, Science 323, 607 (2009).

${ }^{30}$ A. Frøseth, H. Van Swygenhoven, and P. M. Derlet, Acta Mater. 52, 2259 (2004).

${ }^{31}$ H. Van Swygenhoven, P. M. Derlet, and A. G. Froseth, Nat. Mater. 3, 399 (2004).

${ }^{32}$ A. J. Cao and Y. G. Wei, J. Appl. Phys. 102, 083511 (2007).

${ }^{33}$ T. Zhu, J. Li, A. Samanta, H. G. Kim, and S. Suresh, Proc. Natl. Acad. Sci. USA 104, 3031 (2007).

${ }^{34}$ Y. B. Wang, M. L. Sui, and E. Ma, Philos. Mag. Lett. 87, 935 (2007).

${ }^{35}$ Z. H. Jin, P. Gumbsch, K. Albe, E. Ma, K. Lu, H. Gleiter, and H. Hahn, Acta Mater. 56, 1126 (2008).

${ }^{36}$ Y. Kulkarni and R. J. Asaro, Acta Mater. 57, 4835 (2009).

${ }^{37}$ I. Shabib and R. E. Miller, Acta Mater. 57, 4364 (2009).

${ }^{38}$ Z. X. Wu, Y. W. Zhang, and D. J. Srolovitz, Acta Mater. 57, 4508 (2009).

${ }^{39}$ Y. T. Zhu, J. Narayan, J. P. Hirth, S. Mahajan, X. L. Wu, and X. Z. Liao, Acta Mater. 57, 3763 (2009). 
${ }^{40}$ L. Lan and N. M. Ghoniem, Phys. Rev. B 79, 075444 (2009).

${ }^{41}$ X. Y. Li, Y. J. Wei, L. Lu, K. Lu, and H. J. Gao, Nature (London) 464, 877 (2010).

${ }^{42}$ L. Yue, H. Zhang, and D. Y. Li, Acta Mater. 58, 2677 (2010).

${ }^{43}$ A. Stukowski and K. Albe, Phys. Rev. B 82, 224103 (2010).

${ }^{44}$ Y. T. Zhu, X. Z. Liao, and X. L. Wu, Prog. Mater. Sci. 57, 1 (2012).

${ }^{45}$ Y. Mishin, M. J. Mehl, D. A. Papaconstantopoulos, A. F. Voter, and J. D. Kress, Phys. Rev. B 63, 224106 (2001).

${ }^{46}$ H. Tsuzuki, P. S. Branicio, and J. P. Rino, Comput. Phys. Commun. 177, 518 (2007).

${ }^{47}$ J. F. Lutsko, J. Appl. Phys. 65, 2991 (1989).
${ }^{48}$ K. S. Kumar, S. Suresh, M. F. Chisholm, J. A. Horton, and P. Wang, Acta Mater. 51, 387 (2003).

${ }^{49}$ K. S. Kumar, H. Van Swygenhoven, and S. Suresh, Acta Mater. 51, 5743 (2003).

${ }^{50}$ D. Farkas, H. Van Swygenhoven, and P. M. Derlet, Phys. Rev. B 66, 060101(R) (2002).

${ }^{51}$ A. M. Dongare, A. M. Rajendran, B. LaMattina, M. A. Zikry, and D. W. Brenner, Phys. Rev. B 80, 104108 (2009).

${ }^{52}$ I. A. Ovid'ko and A. G. Sheinerman, Phys. Rev. B 77, 054109 (2008).

${ }^{53}$ I. A. Ovid'ko and A. G. Sheinerman, Acta Mater. 52, 1201 (2004). 